\title{
Workload utilization dissemination on grid resources for simulation environment
}

\begin{abstract}
This paper discusses the workload utilization dissemination for grid computing. The CPU is a well-known resource item and it is an integral part in most literatures while other RI's may include memory, network and I/O overhead. The selection of resource variables and the number of RI's involved will result in different definitions of the workload. Various combination of computer RI's have been explored for studying the style of usage, techniques embedded and their capabilities. In contemplating the exploration, this study successfully describe the pattern of workload dissemination through the usage of the RI's and elicited the enhancement factors for systems performance. Among these factors are the manipulation of computer RI's, type of workload information with method of use, the workload dissemination direction along with implementation method and using certain algorithm to come out with new integrated scheduling with load balancing capability. A combination of these factors will help in developing an optimized scheduling or load balancing algorithm.
\end{abstract}

Keyword: Grid; Resource management; Scheduling; Utilization; Load balancing 